\title{
Diacronie
}

Studi di Storia Contemporanea

$\mathrm{N}^{\circ} 32,4 \mid 2017$

Proiezioni individuali e agire collettivo nella storia

\section{Il Pci di Enrico Berlinguer e le elezioni europee attraverso la lettura della stampa comunista (1979-1984)}

\section{Calogero Laneri}

\section{(2) OpenEdition \\ Journals}

Edizione digitale

URL: http://journals.openedition.org/diacronie/6983

DOI: $10.4000 /$ diacronie. 6983

ISSN: 2038-0925

Editore

Association culturelle Diacronie

Notizia bibliografica digitale

Calogero Laneri, « II Pci di Enrico Berlinguer e le elezioni europee attraverso la lettura della stampa comunista (1979-1984) », Diacronie [Online], № 32, 4 | 2017, documento 2, Messo online il 29

décembre 2017, consultato il 30 avril 2019. URL : http://journals.openedition.org/diacronie/6983 ;

DOI : 10.4000/diacronie.6983 


\title{
Diacronie
}

Studi di Storia Contemporanea

\section{$32,4 / 2017$}

Proiezioni individuali e agire collettivo nella storia. Ruoli sociali, aspetti politici e nodi storiografici tra pubblico e privato

\section{Il Pci di Enrico Berlinguer e le elezioni europee attraverso la lettura della stampa comunista (1979-1984)}

\author{
Calogero LANERI
}

Per citare questo articolo:

LANERI, Calogero, «Il Pci di Enrico Berlinguer e le elezioni europee attraverso la lettura della stampa comunista (19791984)», Diacronie. Studi di Storia Contemporanea : Proiezioni individuali e agire collettivo nella storia. Ruoli sociali, aspetti politici e nodi storiografici tra pubblico e privato, 32, 4/2017, 29/12/2017,

URL: <http://www.studistorici.com/2017/12/29/laneri_numero_32/>

Diacronie Studi di Storia Contemporanea $\rightarrow$ http://www.diacronie.it Rivista storica online. Uscita trimestrale.

redazione.diacronie@hotmail.it

Comitato di direzione: Naor Ben-Yehoyada - João Fábio Bertonha - Christopher Denis-Delacour - Maximiliano Fuentes Codera Anders Granås Kjøstvedt - John Paul Newman - Deborah Paci - Niccolò Pianciola - Spyridon Ploumidis - Wilko Graf Von Hardenberg

Comitato di redazione: Jacopo Bassi - Luca Bufarale - Gianluca Canè - Fausto Pietrancosta - Alessandro Salvador - Matteo Tomasoni - Luca Zuccolo.

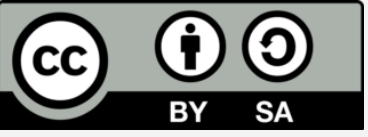

Diritti: gli articoli di Diacronie. Studi di Storia Contemporanea sono pubblicati sotto licenza Creative Commons 3.0. Possono essere riprodotti e modificati a patto di indicare eventuali modifiche dei contenuti, di riconoscere la paternità dell'opera e di condividerla allo stesso modo. La citazione di estratti è comunque sempre autorizzata, nei limiti previsti dalla legge. 


\title{
2/ Il Pci di Enrico Berlinguer e le elezioni europee attraverso la lettura della stampa comunista (1979-1984)
}

\author{
Calogero LANERI
}

Quello tra il Pci e il processo di integrazione comunitaria è un rapporto travagliato che si sviluppa in un vasto arco temporale nel quale il Partito muta integralmente la propria posizione. Nel presente lavoro si intendono prendere in esame le elezioni europee del 1979 e quelle del 1984 poiché è intorno a questo arco temporale, definito da queste due date periodizzanti, che sotto la guida di Enrico Berlinguer ed in virtù del suo impegno diretto nel Parlamento europeo, giunge a compimento la piena e convinta adesione del Pci alle forze dell 'europeismo. L'indagine storica che qui si presenta è stata condotta facendo ricorso alla stampa periodica del Partito e, nello specifico, sono state prese in esame due testate: il settimanale «Rinascita» ed il bimestrale «Critica Marxista».

\section{Quale Europa? Il Pci e le elezioni europee del 1979}

Nella seconda metà degli anni Settanta il Pci guidato da Enrico Berlinguer si configura come un'organizzazione politica che sta abbandonando ogni riserva antieuropeista, che si impegna attivamente all'interno delle istituzioni comunitarie e che lavora - pur non sconfessando l'appartenenza al movimento comunista internazionale - alla costruzione di rapporti privilegiati con le forze socialiste e socialdemocratiche dell'Europa occidentale'. L'impegno europeo, durante gli anni della segreteria Berlinguer, non è uno tra i tanti fronti internazionali, ma ne caratterizza ed influenza tutta l'azione politica ed ideologica, con la definizione, proprio in quegli anni, di un nuovo orizzonte concettuale che pone nuove ed importanti domande a tutto il movimento comunista non soltanto circa il modo di costruire il socialismo ma sulla definizione stessa delle qualità che dovrebbero caratterizzare una futura società socialista ${ }^{2}$.

\footnotetext{
${ }^{1}$ Si veda MAGGIORANI, Mauro, FERRARI, Paolo (a cura di), L'Europa da Togliatti a Berlinguer. Testimonianze e documenti 1945-1984, Bologna, Il Mulino, 2005; FERRARI, Paolo, In cammino verso Occidente. Berlinguer, il PCI e la Comunità europea negli anni '70, Bologna, CLUEB, 2007; DI DONATO, Michele, I comunisti italiani e la sinistra europea. Il PCI e i rapporti con le socialdemocrazie (1964-1984), Roma, Carocci, 2015.

${ }^{2}$ Cfr. PONS, Silvio, Berlinguer e la fine del comunismo, Torino, Einaudi, 2006.
} 
Ed è in questo contesto che matura il lavorio in vista delle prime elezioni europee, che prende corpo già nel novembre del 1978 quando il Centro studi di politica internazionale - in collaborazione con i Gruppi parlamentari del Pci al Senato, alla Camera ed al Parlamento europeo - si fece promotore dell'organizzazione di un convegno dal titolo Quale Europa? I comunisti italiani e le elezioni europee. In tale assise, finalizzata alla definizione della politica europea del Partito in vista della consultazione elettorale, si registrò un vivace dibattito: Spinelli ribadì la necessità che il futuro Parlamento europeo, eletto a suffragio universale, potesse avere un potere costituente ${ }^{3}$ mentre Pajetta si focalizzò sull'azione che la Comunità europea doveva rivestire nell'ambito del rinnovamento delle relazioni internazionali nella prospettiva della distensione e di un nuovo ordine di pace ${ }^{4}$.

Un mese più tardi, l'intervento di Giorgio Amendola al Comitato centrale del Partito segnò l'apertura ufficiale della campagna elettorale: dalle riflessioni del dirigente comunista nacque un libro, I comunisti e le elezioni europee ${ }^{5}$, il secondo testo nel quale Amendola si interroga sui temi della integrazione comunitaria ${ }^{6}$. I numerosi interventi al Comitato centrale di inizio dicembre sancirono la necessità di un impegno intenso in vista delle elezioni europee e proprio la succitata relazione di Amendola rappresentò la base del programma elettorale per il $1979^{7}$.

Senza dubbio alcuno le prime elezioni europee a suffragio universale rivestirono per il Pci un forte valore simbolico, poiché sin dai primi anni Settanta gli europarlamentari comunisti si erano fortemente battuti per l'elezione diretta dei componenti dell'organismo. Pertanto la consultazione venne salutata con grande entusiasmo, nonostante alcune critiche di fondo come la differenziazione della formula elettorale utilizzata dai nove Stati membri. Difatti, contrariamente a quanto chiesto dal Pci, a ciascun paese fu concesso di utilizzare la legge elettorale più affine alle proprie tradizioni ${ }^{8}$.

In Italia la consultazione europea fu oscurata dalle simultanee elezioni politiche che seguirono la caduta del governo della cosiddetta solidarietà nazionale presieduto da Giulio Andreotti: anche dai partiti manifestamente europeisti «un vero interesse per argomenti marcatamente comunitari era rimasto alquanto latente» ${ }^{9}$. Anche per il Pci le elezioni nazionali offuscarono il contestuale

\footnotetext{
${ }^{3}$ CENTRO STUDI PER LA POLITICA INTERNAZIONALE, Quale Europa? I comunisti italiani e le elezioni europee, Roma, Centro studi per la politica internazionale, 1978.

${ }^{4}$ Ibidem.

${ }^{5}$ AMENDOLA, Giorgio, I comunisti e le elezioni europee, Roma, Editori Riuniti, 1979.

${ }^{6}$ Risale ad otto anni prima l'uscita del primo libro del dirigente comunista sulle questioni europee. Cfr. AMENDOLA, Giorgio, I comunisti e l'Europa, Roma, Editori Riuniti, 1971.

${ }^{7}$ «Il dibattito sulla relazione del compagno Giorgio Amendola», in l'Unità, 6 dicembre 1978.

${ }^{8}$ Per la ricostruzione storica del dibattito sulla scelta della legge elettorale si rimanda a PASQUINUCCI, Daniele, Uniti dal voto? Storia delle elezioni europee 1948-2009, Milano, Franco Angeli, 2013, pp. 219-244.

${ }_{9}^{9}$ FLORIS, Doriana, Europei al voto. Politica, propaganda e partecipazione in Italia, Francia e Regno Unito 1979-1989, Milano, Franco Angeli, 2017, p. 83.
} 
impegno europeo, drenando nel Partito molte energie e confinando in secondo piano le elezioni europee che si terranno ad una settimana dalla tornata nazionale ${ }^{10}$.

Benché, dunque, maggiormente impegnato nella consultazione italiana, il Pci animò comunque una vivace campagna elettorale, proponendosi all'elettorato come

forza di cambiamento, capace di innescare un rinnovamento in senso democratico della Comunità, verso una nuova forma di potere in grado di rispondere alle sfide che si ponevano all'Europa, sfide che non erano più sostenibili dai singoli Stati nazionali ${ }^{11}$.

Nei 18 punti del programma elettorale europeo si ritrovano tutti i concetti che Amendola aveva espresso nei suoi contributi degli anni passati e che gli eurodeputati comunisti avevano sviluppato negli anni della partecipazione ai lavori del Parlamento di Strasburgo ${ }^{12}$. Come osservato da Floris la campagna elettorale europea pose tutte le forze politiche di fronte alla necessità di «attuare strategie di partito adatte ad affrontare una disputa nuova, molto più complessa» condizione, da accompagnare necessariamente ad una «maggiore presa di contatto con argomenti poco conosciuti o spesso sottovalutati» ${ }^{13}$. Ed è proprio a queste nuove necessità che risponde il consolidamento dell'interesse dei comunisti italiani nei confronti delle questioni comunitarie: attenzione, quest'ultima, documentata dal costante incremento della produzione di articoli destinati alla stampa periodica del Partito.

Difatti, se agli esordi della partecipazione al Parlamento di Strasburgo la questione europea era trattata dalla stampa comunista alla stregua di tutte le altre vicende internazionali, a partire dal 1979 nella stampa presa in esame lo spazio dedicato al processo di integrazione europea registra una continua crescita. Nell'ambito di questa produzione teorica il 20 aprile, nel n. 15 di «Rinascita», venne pubblicato un articolato supplemento sulle elezioni europee che schematicamente riporta tutte le principali idee dei comunisti italiani sull'Europa.

L'inserto speciale, sulla cui copertina spicca un manifesto elettorale riportante lo slogan Il tuo voto al Pci per un'Europa democratica, si apre con un articolo di Giorgio Amendola. Nell'illustrare i rapporti tra le varie istituzioni della Comunità, il leader comunista denuncia l'aumento di potere del Consiglio dei ministri a scapito del Parlamento, conseguenza, secondo il giudizio di Amendola, «degli egoismi nazionalistici, dei contrasti tra Stati, delle rivalità e dei tentativi di conquistare sulla base dei rapporti di forza posizioni particolari di predominio ${ }^{14}$. In linea con la propria concezione policentrica, per il dirigente comunista la «reale politica di unificazione» può

\footnotetext{
${ }^{10}$ FERRARI, Paolo, op. cit., p. 261.

${ }^{11}$ MAGGIORANI, Mauro, FERRARI, Paolo, op. cit., p. 66.

${ }^{12}$ «Il programma del Pci per le elezioni al Parlamento europeo», in l'Unità, 22 aprile 1979.

${ }^{13}$ FLORIS, Doriana, op. cit., p. 84.

${ }^{14}$ AMENDOLA, Giorgio, «Democrazia e rinnovamento», in Rinascita: Speciale elezioni europee (supplemento), $15 / 1979$, p. 1.
} 
realizzarsi solo con la creazione di un potere multinazionale in grado di frenare egoismi e contrasti tra i singoli Stati appartenenti alla Comunità ${ }^{15}$.

Sull'architettura istituzionale della Comunità europea ritorna anche Luigi Berlinguer, attraverso un contributo mirante ad illustrare il funzionamento della macchina burocratica comunitaria - dai ruoli delle singole istituzioni sino al processo di formazioni delle leggi europee - e le riforme che sarebbero in grado di modificarne la fisionomia, da Berlinguer definita «verticistica e squilibrata» ${ }^{16}$.

Coerentemente con l'azione di democratizzazione e di riduzione dello squilibrio dei poteri tra nazioni, obiettivo da sempre rivendicato dai comunisti italiani, sin dalla prima partecipazione ai lavori del Parlamento europeo gli europarlamentari del Pci si erano resi protagonisti dell'acceso dibattito sulla Politica agricola comune. Nella pubblicazione in esame, il compito di illustrare le proposte comuniste in tema di PAC viene affidato a Luciano Soriente che, in un articolo dall'evocativo titolo «Primo obiettivo è correggere gli squilibri tra i Paesi», critica severamente le politiche agricole comunitarie sottolineando le ingerenze dei paesi più forti e dei gruppi più potenti dell'industria e della finanza ${ }^{17}$.

Tuttavia, se i contributi summenzionati puntano a fornire al lettore analisi approfondite sui temi più stringenti relativi all'attività delle istituzioni comunitarie, è un articolo a firma di Luca Pavolini a restituire un quadro d'insieme contenente, come recita il titolo del pezzo, «Le proposte dei comunisti per un'Europa unita e democratica». La lettura dell'articolo risulta particolarmente interessante poiché il dirigente comunista ripercorre tutti gli aspetti decisivi che qualificano il nuovo intendimento comunista sull'Europa:

Punto di riferimento centrale di questa proposta è l'adozione di una linea di attacco alle pesanti ingiustizie che caratterizzano la società dell'Europa occidentale, all'incertezza del lavoro, alla dura condizione delle masse femminili e giovanili, agli squilibri sociali e territoriali. Questa linea ha come strumento una programmazione democraticamente elaborata e controllata che abbia l'obiettivo di un uso razionale di una giusta ripartizione delle risorse, ponendo fine all'anarchia e agli sprechi che contraddistinguono la situazione attuale, la concorrenza sfrenata, la corsa al massimo profitto, la tendenza al formarsi di posizioni monopolistiche ${ }^{18}$.

Il tema dell'unità europea risulta dunque centrale nella proposta programmatica dei comunisti italiani e la spinosa dicotomia tra costruzione comunitaria e ruolo dello Stato nazione non poteva

\footnotetext{
${ }^{15}$ Ibidem, pp. 2-3.

${ }^{16}$ BERLINGUER, Luigi, «Le istituzioni europee», in Rinascita: Speciale elezioni europee (supplemento), 15/1979, pp. 6-7.

${ }^{17}$ SORIENTE, Luciano, «Primo obiettivo è correggere gli squilibri tra i Paesi», in Rinascita: Speciale elezioni europee (supplemento), 15/1979, p. 12.

${ }^{18}$ Ibidem, p. 20.
} 
che essere affidata ad Altiero Spinelli, personificazione del nuovo corso comunista sull'Europa. A tal proposito appare qui doveroso fare cenno al processo di riavvicinamento tra il leader federalista e il Pci che trovò il proprio culmine nella candidatura in occasione della tornata elettorale del 1976. Proveniente da una giovanile adesione al comunismo, durante il fascismo il futuro leader federalista aveva scontato un lungo periodo di confino nel quale si produsse un allontanamento dal Pci che portò alla sua espulsione dalle fila del Partito ${ }^{19}$. Tuttavia, a partire dal 1972, le pagine dei suoi diari testimoniano lo svilupparsi di una forte curiosità nei confronti della revisione del Pci sui temi comunitari ${ }^{20}$. Difatti, se il rapporto tra Spinelli e il variegato mondo della sinistra europea rappresentò un «segmento importante della vicenda politica del federalista italiano ${ }^{21}$, in questa fase Spinelli trova nel Partito di Berlinguer l'unico partito comunista occidentale che sceglie di inserire la dimensione comunitaria nella propria strategia teorica ${ }^{22}$. Il riavvicinamento è determinato inoltre da riflessioni più generali non direttamente collegate alla nuova linea dei comunisti italiani sull'Europa, come la crescente delusione del leader federalista nei confronti della Commissione ${ }^{23}$. È da queste premesse che, nel 1976, il lento riavvicinamento tra il Pci ed Altiero Spinelli giunse a compimento quando il Partito, alla ricerca di indipendenti di sinistra da inserire nelle proprie liste in occasione delle imminenti elezioni politiche, offre e Spinelli la candidatura con la promessa che sarebbe stato indicato dal Partito al seguente rinnovo della rappresentanza italiana al Parlamento europeo ${ }^{24}$. Spinelli accetta la proposta annunciando parallelamente la decisione di autosospendersi dai lavori della Commissione, specificando l'intenzione di dimettersi in caso di elezione. Tre anni dopo, in ottemperanza a quanto concordato, Spinelli venne candidato nelle liste del Pci consegnando al Partito una forte carica legittimatrice. Purtuttavia, come sottolinea Pasquinucci, la riflessione sull'inserimento di Spinelli nelle liste comuniste non può esaurirsi in una mero calcolo del «grado di europeizzazione» del Pci ma va contestualizzata nella più vasta pratica comunista di coinvolgimento degli intellettuali da un lato e l'inserimento del Partito nel «gioco democratico e nazionale» dall'altro ${ }^{25}$.

Spinelli contribuì alla stesura dell'opuscolo qui in esame con un articolo dal titolo «Stati, nazioni e popoli di fronte all'idea di Europa» nel quale vengono analizzate le criticità all'interno di Francia, Inghilterra e Danimarca, gruppo di Paesi nei quali, secondo l'autore dell'articolo, «l'idea dell'unità europea è un'idea che divide gli animi, perché nella misura in cui la sua realizzazione limita i poteri dello Stato, essa è facilmente sentita come limitante della vita

\footnotetext{
${ }^{19}$ GRAGLIA, Piero, Altiero Spinelli, Bologna, Il Mulino, 2008, pp. 113-121.

${ }^{20}$ Cfr. SPINELLI, Altiero, Diario europeo 1970-1976, Bologna, Il Mulino, 1991. Tra le altre, rappresentative di questo crescente interesse sono le annotazioni del 2 dicembre 1972, del 14 febbraio 1973, del 13 settembre 1974 e del 1 dicembre 1975.

${ }^{21}$ PASQUINUCCI, Daniele, Europeismo e democrazia. Altiero Spinelli e la sinistra europea 1950-1986, Bologna, Il Mulino, 2000, p. 9.

${ }^{22}$ FERRARI, Paolo, op. cit., p. 214.

${ }^{23}$ Ibidem, p. 215.

${ }^{24}$ I dettagli della candidatura vengono narrati dall'interessato in SPINELLI, Altiero, op. cit., pp. $940-941$.

${ }^{25}$ PASQUINUCCI, Daniele, Europeismo e democrazia. Altiero Spinelli e la sinistra europea 1950-1986, cit., p. 302.
} 
nazionale, e suscita reazioni negative» ${ }^{26}$. L'articolo di Spinelli è seguito da un contributo a firma di Bruno Ferrero nel quale viene sviluppato uno dei temi portanti di tutta la riflessione europeista del Pci, ovvero la lotta alle diseguaglianze tra i Paesi membri della Comunità. Per Ferrero «le aree forti del centro Europa si integrano fra di loro e si collegano con le nuove vie commerciali e con i nuovi mercati. Le aree deboli del Mediterraneo, per evitare la più completa emarginazione, possono, al massimo, ambire a rincorrere le prime» ${ }^{27}$.

Le elezioni europee, nella narrazione elettorale del Pci, rappresentano dunque uno strumento in grado di delineare nuovi rapporti di forza all'interno delle istituzioni comunitarie, con il dichiarato obiettivo di «segnare una svolta positiva ed importante nelle linee di politica economica e sociale» ${ }^{28}$. In tal senso è proprio la posizione sul governo dell'economia comunitaria che ci fornisce la più originale delle elaborazioni dell'europeismo del Pci: se da un lato viene aspramente criticata la linea economica comunitaria - ritenuta limitativa, residuale e marginale ${ }^{29}$ - dall'altro il Partito si rifiuta di attribuire al primato degli Stati nazionali sulle istituzioni comunitarie tutte le colpe di tali insufficienze. Difatti, secondo il giudizio dei comunisti italiani, limiti ed incertezze non derivano dall'eccesso di potere degli Stati, bensì dalla mancanza di contributo di questi ultimi alla costruzione di momenti di reale governo dell'economia a più livelli: «sovranazionale, nazionale e regionale». L'eclettico giudizio in tema di governance economica europea conferma come la revisione delle posizioni antieuropeiste da parte del gruppo dirigente comunista non si prefigurò come un processo lineare ma come un lungo percorso nel quale, ancora in questa fase, permangono contraddizioni di fondo che tradiscono le difficoltà dei comunisti italiani nell'inserire la linea europeista in una coerente visione delle relazioni internazionali ${ }^{30}$.

A tal proposito assai emblematica appare la vicenda del dibattito parlamentare sull'adesione dell'Italia al Sistema monetario europeo svoltosi nel dicembre del 1978, a pochi mesi dal voto europeo. La discussione vide entrare in conflitto le posizioni su politica italiana ed europea poiché i comunisti italiani percepirono nello Sme un impedimento alla promozione delle riforme economiche italiane che la coalizione di solidarietà nazionale avrebbe dovuto varare ${ }^{31}$. In sede parlamentare la posizione di bocciatura del Pci fu espressa da Giorgio Napolitano che, pur sostenendo come l'europeismo del proprio partito fosse ormai comprovato ${ }^{32}$, dichiarò che un

\footnotetext{
${ }^{26}$ SPINELLI, Altiero, «Stati, nazioni e popoli di fronte all'idea di Europa», in Rinascita: Speciale elezioni europee (supplemento) 15/1979, p. 18.

${ }^{27}$ FERRERO, Bruno, «Il rischio della "doppia velocità"», in Rinascita: Speciale elezioni europee (supplemento), 15/1979, p. 24.

${ }^{28}$ Ibidem.

${ }^{29}$ Ibidem, p. 25.

${ }^{30}$ CRUCIANI, Sante, Le sinistre italiane e l'Europa da Togliatti e Nenni al Partito Democratico, in ID. (a cura di), Il socialismo europeo e il processo di integrazione. Dai Trattati di Roma alla crisi politica dell'Unione (1957-2016), Milano, Franco Angeli, 2016, p. 41.

${ }^{31}$ MAGGIORANI, Mauro, FERRARI, Paolo, op. cit., p. 70.

${ }^{32}$ CAMERA DEI DEPUTATI, VII Legislatura, Resoconto Stenografico, 12 dicembre 1978, p. 24993.
} 
ingresso immediato nello Sme avrebbe comportato conseguenze negative per il Paese in termini di ristagno "della produzione, degli investimenti e dell'occupazione»" ${ }^{33}$. La bocciatura dello Sme da parte del Pci determinò altresì una radicale divergenza di vedute con Altiero Spinelli, ponendo in evidenza la complessità del rapporto tra il Partito e il leader federalista ${ }^{34}$. Ai fini della nostra riflessione è tuttavia indicativo come il Partito nel motivare il proprio voto negativo, alla vigilia delle prime elezioni europee, tenne a precisare che l'opposizione allo Sme non rappresentava un voto contro l'Europa poiché «l'interesse del nostro paese, e specificamente l'interesse del nostro Mezzogiorno» afferma Napolitano in sede di discussione parlamentare «coincide con la causa di uno sviluppo della Comunità» ${ }^{35}$.

L'articolato inserto mediante il quale il Partito presenta agli elettori la propria proposta programmatica è chiuso da un contributo di Nilde Iotti. Nella riflessione della dirigente comunista emergono con più chiarezza le considerazioni sull'Europa come uno spazio geografico autonomo ed indipendente:

Noi respingiamo l'idea che il processo di integrazione economica e politica europea - di cui il Parlamento europeo eletto è senza alcun dubbio un momento non trascurabile - significhi al tempo stesso la creazione di un altro polo armato. Questo non rafforzerebbe le vie della pace. $\mathrm{Al}$ contrario. Significherebbe rendere insostenibile la tensione mondiale, perché è certo che sia gli Stati uniti sia l'Unione Sovietica non potrebbero tollerare un così profondo mutamento negli equilibri del mondo ${ }^{36}$.

Viene sviluppata, quindi, l'originale formula di un'Europa né antisovietica né antiamericana ipotizzata da Enrico Berlinguer sei anni prima, sostenendo che tale prospettiva appare realizzabile solamente mediante l'apporto dei comunisti all'interno delle istituzioni comunitarie ${ }^{37}$.

Nonostante l'impegno profuso, il 10 giugno, giorno della consultazione elettorale, il risultato restituito dalle urne non può che deludere i dirigenti comunisti. Il Partito conquista il 29,5\%, scendendo ulteriormente rispetto al già insoddisfacente $30,4 \%$ raccolto alle politiche della settimana precedente. Sull'erosione di consensi pesa indubbiamente il giudizio negativo sull'esperienza della solidarietà nazionale ${ }^{38}$.

L'influenza delle dinamiche nazionali sul voto europeo appare evidente: i risultati elettorali esprimono la congiuntura politica presente all'interno dei singoli Stati generando addirittura la

\footnotetext{
${ }^{33}$ Ibidem, p. 24995.

${ }^{34}$ Per Spinelli lo Sme rappresentava un simbolo del predominio della dimensione intergovernativa su quella sovranazionale poiché lasciava la politica economica e monetaria nelle mani dei singoli Paesi. Cfr. PASQUINUCCI, Daniele, Europeismo e democrazia. Altiero Spinelli e la sinistra europea 1950-1986, cit., pp. 305-308.

${ }^{35}$ CAMERA DEI DEPUTATI, VII Legislatura, Resoconto Stenografico, 12 dicembre 1978, p. 24998.

${ }^{36}$ IOTTI, Nilde, «Autonomia e policentrismo per vincere l'equilibrio del terrore», in Rinascita: Speciale elezioni europee (supplemento), 15/1979, p. 31.

${ }^{37}$ Ibidem, p. 32.

${ }^{38}$ FERRARI, Paolo, op. cit., p. 272.
} 
sensazione che non solo il nuovo Parlamento eletto non abbia conquistato l'auspicato consolidamento ma che esso sia addirittura meno rappresentativo della precedente Assemblea ${ }^{39}$. Il Pci porta a Strasburgo ventiquattro degli ottantuno eurodeputati eletti in Italia che costituiranno, insieme a diciannove francesi ed un danese, il gruppo dei comunisti e apparentati ${ }^{40}$.

Il lavoro della rappresentanza comunista a Strasburgo rafforzò ancor di più l'idea della Comunità europea come teatro politico più idoneo nel quale condurre importanti battaglie politiche dal respiro internazionale capaci di rilanciare la politica estera del Pci. Il gruppo dirigente comunista era infatti consapevole che, dopo il vertice fra i partiti comunisti di Italia, Francia e Spagna tenuto a Madrid nel marzo del 1977, il percorso dell'eurocomunismo aveva imboccato una parabola discendente ${ }^{41}$. In base a tale visione il Partito delineò un nuovo internazionalismo capace di mettere in relazione tutte le forze della sinistra, con l'obbiettivo di approfondire il ruolo chiave dell'Europa nel rapporto con le due superpotenze, con i movimenti di liberazione e con i Paesi nati dal lungo processo di decolonizzazione. Difatti, malgrado l'acuirsi della crisi nel rapporto tra Pci e Psi ${ }^{42}$, fuori dai confini nazionali si consolidò la tendenza dei comunisti italiani a collegare le proprie posizioni a quelle espresse dai socialdemocratici dell'Europa del Nord ${ }^{43}$.

È in quest'ottica che si inserisce l'impegno del Pci nella battaglia per lo smantellamento delle basi militari grazie alla quale si sviluppò quell'humus teorico con il quale il Partito criticherà severamente la decisione sovietica di intervenire militarmente in Afghanistan ${ }^{44}$, facendo propria una posizione in aperto contrasto con la linea sovietica ${ }^{45}$. Appare qui interessante sottolineare come, a conferma di quanto affermato in precedenza, sul tema si concretizzò un forte dialogo e una certa convergenza degli oratori delle sinistre: il documento presentato dal Pci raccolse i voti dei comunisti e apparentati italiani e di alcuni deputati di altri gruppi, mentre l'insieme del gruppo socialista si astenne. Analogamente comunisti e socialisti votarono insieme contro il

\footnotetext{
${ }^{39}$ FLORIS, Doriana, op. cit., p. 111.

${ }^{40}$ Ibidem.

${ }^{41}$ DI MAGGIO, Marco, Alla ricerca della terza via al socialismo. I PC italiano e francese nella crisi del comunismo (1964-1984), Napoli-Roma, Edizioni scientifiche italiane, 2014, pp. 311-317.

${ }^{42}$ Per la ricostruzione del rapporto tra Enrico Berlinguer e Bettino Craxi tra il 1979 e il 1984 si rimanda a KARRER, Livio, "Dopo la "solidarietà nazionale". Comunisti e socialisti tra anni Settanta e Ottanta», in Dimensioni e problemi della ricerca storica, 2/2016, pp. 95-119.

${ }^{43}$ DI DONATO, Michele, I comunisti italiani e la sinistra europea. Il PCI e i rapporti con le socialdemocrazie (19641984), cit., p. 255.

${ }^{44}$ Emblematica la dichiarazione pronunciata da Berlinguer al Parlamento europeo in occasione della discussione sull'intervento sovietico in Afghanistan. Si veda CECCHETELLI, Patrizia, MIGLIARDI, Giorgio, MUGNAI, Sergio (a cura di), Berlinguer parlamentare europeo, Roma, Salemi, 1984, pp. 17-21.

${ }^{45}$ Nonostante la crisi nei rapporti tra Botteghe Oscure e Mosca, è intorno al tema della capacità dell'Unione Sovietica di costituire una minaccia internazionale che si situa, ancora in questa fase, una delle principali divergenze tra il Partito di Berlinguer e la sinistra europea. Cfr. PONS, Silvio, Il socialismo europeo, la sinistra italiana e la crisi del comunismo, in COLARIZI, Simona, CRAVERI, Piero, PONS, Silvio, QUAGLIARIELLO, Gaetano (a cura di), Gli anni ottanta come storia, Soveria Mannelli, Rubbettino, 2004, pp. 215-230 (p. 226).
} 
documento presentato dalla maggioranza di centro-destra dell'Assemblea ${ }^{46}$.

Parallelamente, se il conflitto afghano aveva determinato la più rilevante crisi dei rapporti tra comunisti italiani ed Unione Sovietica durante la fase della cosiddetta seconda guerra fredda ${ }^{47}$, la piena adesione del Pci al Progetto Spinelli rappresentò il più emblematico sostegno non solo ai disegni di integrazione già in atto ma ad un piano ancora più innovatore in tema di attribuzione di poteri ad una realtà sovranazionale ${ }^{48}$. Infatti, con una radicalità alla quale non aveva mai fatto ricorso, Berlinguer, in occasione della seduta del 13 settembre 1983, motivando la propria adesione al Progetto affermava:

La dimensione comunitaria è quella adeguata per far fronte con una vera forza economica, politica e culturale alle sfide e alle trasformazioni del nostro tempo. [...] Da ciò deriva, secondo noi, che vanno posti in modo nuovo - diverso dal passato - i tradizionali termini del contrasto sovrannazionalità - difesa degli interessi nazionali. [...] Andare su questa strada significa dunque rispondere con lungimiranza agli interessi veri dei nostri popoli ${ }^{49}$.

È l'acquisizione, irrevocabile, di una nuova teoria per l'Europa e sull'Europa.

\section{L'europeismo comunista alla prova del voto: le elezioni dello storico «sorpasso»}

Le elezioni europee del 1984, le seconde della storia della Comunità, si svolsero il 17 giugno. Rispetto alla precedente consultazione, in questa tornata erano chiamati a votare anche gli elettori greci ${ }^{50}$. All'appuntamento elettorale è chiamata un'Italia che negli anni Ottanta aveva puntato ad estendere lo spazio in cui la propria politica estera potesse trovare espressione: si pensi al dibattuto tema degli euromissili, agli accordi israelo-egiziani e alla missione di pace in Libano $^{51}$.

Il Partito comunista italiano si presentò all'elettorato carico di quel patrimonio europeista accresciutosi durante l'esperienza al Parlamento europeo ed il rinnovato quadro teorico-politico

\footnotetext{
${ }^{46}$ CECCHETELLI, Patrizia, MIGLIARDI, Giorgio, MUGNAI, Sergio (a cura di), op. cit., p. 17.

${ }^{47}$ DI MAGGIO, Marco, «Internazionalismo, socialismo ed europeismo nel Pci di Berlinguer», in Dimensioni e problemi della ricerca storica, 2/2016, pp. 55-78 (p. 72).

${ }^{48}$ Sul rapporto tra Berlinguer ed Altiero Spinelli cfr. HÖBEL, Alexander, Enrico Berlinguer parlamentare europeo. Il dialogo con Altiero Spinelli, in CIOFI, Paolo, LOPEZ, Gennaro (a cura di), Berlinguer e l'Europa. I fondamenti di un nuovo socialismo, Roma, Editori Riuniti, 2016, pp. 53-68.

${ }_{49}$ CECCHETELLI, Patrizia, MIGLIARDI, Giorgio, MUGNAI, Sergio (a cura di), op. cit., p. 42.

${ }^{50}$ L'ingresso della Grecia nella Comunità Europea del 1981 era stato fortemente sostenuto dal Pci sin dalla fine degli anni Settanta. Cfr. ORILIA, Vittorio, «I comunisti italiani, la Grecia e l'Europa», in Rinascita, 2/1979, p. 33.

${ }^{51}$ FLORIS, Doriana, op. cit., p. 132.
} 
sull'Europa venne presentato dallo stesso Enrico Berlinguer in una lunga intervista rilasciata a «Critica Marxista» agli inizi del 1984. Qui, come in ogni altra riflessione introduttiva sulla questione europea, il segretario comunista esprime la propria critica per l'immobilismo che caratterizza le istituzioni comunitarie riferendosi, in particolare, alle deludenti riunioni del Consiglio europeo del dicembre 1983 ad Atene e del marzo del 1984 a Bruxelles ${ }^{52}$. Se per Berlinguer la crisi delle istituzioni comunitarie si configura come un dato oggettivo della realtà, nel giudizio del dirigente comunista la soluzione di tale stallo non può tuttavia consistere nel riemergere di posizioni nazionalistiche.

Ora, lo spirito nazionale, il senso della nazionalità è certamente, rispetto a quello internazionale e internazionalista, una realtà più corposa e radicata di quanto Marx e la stessa tradizione socialista abbiano ritenuto. Non è pensabile che la via d'uscita dalla crisi della Comunità europea possa consistere nel ripiegamento di ogni singolo Stato sulla sua peculiare identità, nel rinchiudersi nelle particolarità dei propri interessi ${ }^{53}$.

In tale analisi il tema della cooperazione tra le nazioni assume ancora una volta valore centrale e, interrogato dall'intervistatore sui «contrasti che paralizzano l'azione della stessa Comunità» e sulla «impotenza del parlamento eletto del 1979», Berlinguer ribadisce il concetto secondo il quale la crisi di legittimità che ha colpito il processo comunitario non può essere superata mettendo in discussione la necessità del progetto stesso bensì assegnando alle istituzioni europee quei poteri che possano affermare un progressivo primato del metodo comunitario su quello intergovernativo ${ }^{54}$.

La richiesta di un maggior potere nelle mani dell'Assemblea di Strasburgo esorta l'intervistatore a ricordare l'antieuropeismo comunista delle origini e di come parte delle contraddizioni su cui si fondava la Comunità permangono ancora. Sul tema il segretario comunista non nega la legittimità della scelta antieuropeista dei primi anni Cinquanta, sottolineando come allora tale scelta fosse motivata dalla necessità di respingere quei progetti che vedevano nella sovranazionalità uno «strumento per far arretrare il movimento operaio dei paesi nei quali, come in Italia, esso era più avanzato ${ }^{55}$. Tuttavia, secondo la riflessione di Berlinguer, il disegno liberista delle origini attraversa oggi una profonda crisi e la stessa Comunità «ha finito con il ridursi a garantire alcuni elementi di libera circolazione delle merci e degli uomini».

\footnotetext{
${ }^{52}$ ZANARDO, Aldo, «L'Europa, la pace, lo sviluppo. Intervista ad Enrico Berlinguer», in Critica Marxista, 12/1984, p. 6.

${ }^{53}$ Ibidem.

${ }^{54}$ Ibidem, pp. 6-7.

${ }^{55}$ Ibidem, p. 7.
} 
Ciononostante il progetto comunitario ha alimentato nei popoli europei, nelle forze sociali, culturali e politiche più avvertite la consapevolezza dell'«oggettivo bisogno di Europa» ${ }^{56}$. La flessione del dato dell'affluenza alle elezioni del 1984, un elemento che negli anni diventerà una costante del voto europeo, sconfesserà la troppo ottimistica riflessione del segretario comunista $^{57}$.

In merito alla crisi economica internazionale e all'inadeguatezza delle istituzioni comunitarie nel fornire una risposta ai problemi della crescita economica, Berlinguer afferma che il sottosviluppo del sud del pianeta è strettamente correlato alla realtà del nord giacché

l'umanità occidentale ha impostato per secoli il suo rapporto con l'altra umanità in termini di interesse puramente immediato, di colonizzazione o dominazione, di rapina delle risorse, addirittura di schiavizzazione e non in termini di uguaglianza e di promozione ${ }^{58}$.

Nella proposta europeista di Berlinguer l'Europa ricopre un ruolo di soggetto forte ed autonomo delle relazioni internazionali, investito dunque della funzione storica di limitare le differenze sociali ed economiche tra le nazioni. Tuttavia per il segretario comunista ciò non sarà realizzabile fin quando l'Europa «non si deciderà ad affrontare il tema del sottosviluppo del terzo e quarto mondo e, anche, dei suoi rapporti con l'est ${ }^{59}$.

L'autore dell'intervista coglie questo ultimo spunto finale per approfondire il tema dei rapporti con il blocco sovietico. Berlinguer, il leader comunista dello strappo, rimarca le criticità in cui verte il sistema socialista sia nella sfera della partecipazione democratica che in quella della economia.

Da un tempo non breve ci troviamo a constatare che nei paesi dell'est i ritmi di crescita economica, e anche tecnologica e scientifica, sono piuttosto contenuti. È vero che, di questi paesi, si sottolineano prevalentemente le insufficienze della vita politica e sociale, la non crescita o la crescita non significativa della democrazia politica. [...] Questa crisi deriva anche, e ampiamente, da insufficienze intrinseche alle strutture istituzionali di quei paesi ${ }^{60}$.

Il commento di Berlinguer conferma l'ormai diffusa percezione occidentale della crisi del comunismo sovietico e della conseguente necessità di costruire intorno al tema dell'Europa - e dei rapporti con le forze della famiglia socialista europea - parte della più complessiva analisi

\footnotetext{
${ }^{56}$ Ibidem, pp. 7-8.

${ }^{57}$ PASQUINUCCI, Daniele, Uniti dal voto? Storia delle elezioni europee 1948-2009, cit., p. 308.

${ }^{58}$ Ibidem.

${ }^{59}$ Ibidem, pp. 8-9.

${ }^{60}$ Ibidem, pp. 9-10.
} 
berlingueriana delle relazioni internazionali che, nella fase successiva al crollo del Muro di Berlino, favorirà il graduale ricollocamento politico e culturale del Partito ${ }^{61}$.

Tuttavia, ciò che qui appare interessante sottolineare è come Berlinguer, nell'affrontare i nodi spinosi della costruzione comunitaria, capovolga totalmente la vecchia narrazione che aveva caratterizzato l'antieuropeismo comunista degli anni Cinquanta, dichiarando che l'impasse nella quale si trova l'Europa è provocata da gruppi conservatori presenti in larga misura nei paesi nordeuropei, i quali ritengono che una via comunitaria dello sviluppo sia impraticabile a causa del divario incolmabile tra nord e sud della Comunità ${ }^{62}$. L'affondo di Berlinguer è radicale: «[...] una crescita realmente grande, non condizionata e non subalterna dello stesso nord dell'Europa, può aversi solo nel quadro di una integrazione europea ${ }^{63}$.

Intervenendo poi sulla questione della pace, tema preminente nella narrazione berlingueriana, il politico sardo sottolinea come la crisi dei rapporti internazionali possa superarsi solo mediante una politica di disarmo rappresentante l'«unica risposta sicura alla ricerca di sicurezza». La proposta centrale in tema di sicurezza rimane dunque il blocco degli armamenti ed una riduzione «progressiva, bilanciata, controllata e controllabile» di armi nucleari, non convenzionali e chimiche attualmente nelle disponibilità di entrambe le superpotenze.

L'Europa unita e autonoma, potrebbe essere decisiva. Ogni stato europeo è in grado di promuovere, anche da solo, iniziative per la distensione e il disarmo: ma nessuno Stato europeo, da solo, ha una facoltà di incidere pari a quella che avrebbe la Comunità nel suo insieme ${ }^{64}$.

Le possibilità offerte dal processo di distensione rappresentano dunque uno degli assi portanti non solo della posizione berlingueriana sul processo comunitario, ma divengono altresì terreno di confronto tra il Pci e le sinistre europee ${ }^{65}$. Tuttavia tale posizione non appare priva di contraddizioni e, incalzato sulle criticità di un'autonomia «inerme», Berlinguer sottolinea che

Le forze politiche le quali sostengono che la difesa dell'Europa va cercata nella formazione di un terzo blocco militare si mettono in una posizione senza via di uscita. [...] L'Europa è come costretta, per sue intrinseche ragioni non solo economiche, ad una politica di pace. A costringerla c'è il fatto che l'Europa è, tra ovest ed est, territorio di confine e incrocio ${ }^{66}$.

\footnotetext{
${ }^{61}$ PONS, Silvio, Il socialismo europeo, la sinistra italiana e la crisi del comunismo, in COLARIZI, Simona, CRAVERI, Piero, PONS, Silvio, QUAGLIARIELLO, Gaetano (a cura di), Gli anni ottanta come storia, cit., pp. 215-230 (p. 215).

${ }^{62}$ Ibidem.

${ }^{63}$ Ibidem, pp. 12-13.

${ }^{64}$ Ibidem, p. 14.

${ }^{65}$ DI DONATO, Michele, «Enrico Berlinguer e la sinistra europea», in Dimensioni e problemi della ricerca storica, 2/2016, pp. 37-54 (p. 40).

${ }^{66}$ ZANARDO, Aldo, «L'Europa, la pace, lo sviluppo. Intervista ad Enrico Berlinguer», in Critica Marxista, 12/1984, p. 15.
} 
Alle critiche del disegno di disarmo berlingueriano, spesso tacciato di impraticabile idealismo, il segretario risponde evidenziando i rischi di un'ipotetica formazione di un terzo blocco militare:

[...] se l'Europa prendesse la via di divenire un terzo blocco militare, la direzione della vita politica europea finirebbe per essere presa, prima o poi, da gruppi e caste reazionarie. [...] Abbiamo anzitutto la pressione degli Stati Uniti. Questo Paese ha una consistenza politica, militare ed economica immensa e tende manifestamente, con il peso che gli proviene da questa forza, a impostare i rapporti con l'occidente europeo in termini di subalternità ${ }^{67}$.

Nella domanda conclusiva con la quale si chiude l'intervista, Berlinguer ritorna nuovamente sulle difformità di giudizio sull'Europa tra il Pci ed una larga parte delle forze operaie, socialiste e popolari che non ritengono che la sfida dell'integrazione europea risponda ai propri interessi. Il segretario del Pci rilancia la necessità di condurre efficientemente le lotte per un nuovo ordine internazionale costituito da società meno ingiuste, paragonando l'integrazione comunitaria all'Unità d'Italia che, nel giudizio del leader comunista, "costituì un enorme progresso e creò al tempo stesso condizioni più favorevoli per l'ascesa delle masse popolari» ${ }^{68}$.

Com'è noto l'accesa campagna elettorale per le elezioni europee si interruppe violentemente a Padova il 7 giugno, con il malore e la successiva morte di Enrico Berlinguer ${ }^{69}$. Il segretario comunista si spense infatti quattro giorni dopo ed il Pci perdeva, oltre al leader indiscusso di una lunga stagione, uno degli esponenti politici italiani più appassionati alle grandi questioni europee ed internazionali ${ }^{70}$.

La morte di Enrico Berlinguer colpì profondamente tutto il popolo comunista, che il 13 giugno si radunò per l'ultimo ed imponente saluto al proprio segretario ${ }^{71}$. Quattro giorni dopo i partecipati funerali, le operazioni di spoglio delle consultazioni europee restituirono un dato clamoroso: per la prima volta il Partito comunista italiano si attestò come primo partito, superando, seppur di poco, la Democrazia cristiana ${ }^{72}$.

Il numero di «Rinascita» chiuso in tipografia la mattina del 19 giugno riportò in copertina una raffigurazione del volto di Berlinguer su di una folla sterminata. Sulla evocativa rappresentazione campeggia l'inequivocabile titolo il cui senso starà alla base dell'analisi del voto: «Ha vinto il Partito di Enrico Berlinguer». Il ricordo del segretario scomparso anticipa l'analisi del risultato

\footnotetext{
${ }^{67}$ Ibidem, p. 16

${ }^{68}$ Ibidem, p. 18.

${ }^{69}$ KARRER, Livio, «Una difficile traslazione. I funerali di Palmiro Togliatti e di Enrico Berlinguer», in Dimensioni e problemi della ricerca storica, 2/2011, pp. 109-143 (p. 121).

${ }^{70}$ MAGGIORANI, Mauro, FERRARI, Paolo, op. cit., p. 75

${ }^{71}$ VITTORIA, Albertina, Storia del Pci 1921-1991, Roma, Carocci, 2000, p. 147.

${ }^{72}$ FLORIS, Doriana, op. cit., p. 158.
} 
elettorale, attraverso undici pagine nelle quali vengono riproposte tutte «le tappe di una grande politica».

Il lungo encomio è seguito dal Dossier elezioni, nel quale viene analizzato il risultato elettorale in ogni suo aspetto, nazionale ed internazionale.

Nel primo contributo, il cui autore è Mario Tronti, si sostiene che il voto europeo non possa essere analizzato come un voto omogeneo poiché la stessa Europa politica non è ancora una realtà uniforme. Per il filosofo il voto non ha restituito indicazioni univoche anche a causa della genericità dell'azione della Comunità sui grandi temi, primo su tutti quello della politica estera. «La politica estera» scrive Tronti «rimane privilegio dei governi. Si può intervenire dal basso, ma per condizionare e orientare, non per risolvere e decidere. La dialettica movimenti-istituzioni è più debole nell'Europa comunitaria che in ogni Stato nazionale» ${ }^{73}$. Per queste ragioni, secondo il giudizio del filosofo, l'aspetto più interessante offerto dal risultato elettorale è rappresentato dalla tradizionale distinzione destra-sinistra che si ripresenta sotto forma di distinzione tra governo e opposizione. «Le pratiche governative» sostiene l'autore dell'articolo «si somigliano tutte, e forse proprio per questo nessuna riesce a ricevere un consenso stabile» ${ }^{74}$. Per Tronti, di conseguenza, la vecchia Europa non riesce a trovare un modello di governo per il «capitalismo maturo» e ciò ha determinato l'abbandono dell'idea di "governo contro le masse» in favore dell'idea moderata di «governo senza le masse». Questo meccanismo, secondo l'analisi dell'intellettuale comunista, genera un forte rifiuto per lo status quo, una «stanchezza e noia per il già visto, una volontà di cambiamento che si esprime in mille contraddittori modi» e che favorisce l'ascesa di masse senza classi «che non trovano, e non cercano, nomenclature di partito» ${ }^{75}$. A giudizio dello scrivente, tra tutte quelle pubblicate nel dossier, l'analisi di Tronti spicca per una lucidità la cui lettura postuma appare quasi profetica.

Se l'oggetto dell'analisi di Mario Tronti è rappresentato dalla crisi delle forze governative in favore di quelle di opposizione, Massimo Ghiara focalizza invece la propria analisi sul sorpasso del Pci sulla Dc. Per Ghiara le urne hanno consegnato un risultato perentorio, difficilmente prestabile a sottigliezze e sfumature: «L'incontestabile vittoria del Pci» sostiene «è una vittoria che sanziona una situazione senza precedenti storici in Italia» ${ }^{76}$. Nell'articolo vengono analizzati in dettaglio i dati delle cinque circoscrizioni, giungendo alla conclusione che l'attuale maggioranza che governa il Paese è «prossima alla dissoluzione» ${ }^{77}$. I giudizi politici espressi da Ghiara appaiono estremamente suggestionati da un comprensibile ricorso ad una dialettica propagandistica che verrà tuttavia smentita dalla crisi che il Pci vivrà negli anni successivi. Difatti, benché le elezioni europee del 1984 abbiano segnato lo storico sorpasso del Pci sulla Dc, è possibile affermare come,

\footnotetext{
${ }^{73}$ TRONTI, Mario, «L'Europa delle trasformazioni», in Rinascita, 25/1984, p. 25.

${ }^{74}$ Ibidem.

${ }^{75}$ Ibidem

${ }^{76}$ GHIARA, Massimo, «Come è avvenuto il sorpasso», in Rinascita, 25/1984, p. 26.

${ }^{77}$ Ibidem, p. 27.
} 
al contempo, l'esito elettorale fuorviò i dirigenti del Partito in merito alla necessità di elaborare una nuova strategia teorica capace di confrontarsi con i moderni paradigmi culturali che stavano attraverso la società dell'epoca ${ }^{78}$.

Se Ghiara si focalizza sugli aspetti nazionali del risultato elettorale, Claudio Petruccioli, al contrario, esamina il voto fuori dagli schematismi statali. Il dirigente comunista sviluppa la propria valutazione partendo da una riflessione introduttiva secondo la quale il richiamo alle urne, per la seconda volta dopo il 1979, rappresenta già di per sé un dato positivo, tenuto conto degli esiti fallimentari dei recenti vertici europei. Petruccioli, in linea con uno dei capisaldi della narrazione europeista del Pci, sottolinea come il processo della unità europea può trovare la spinta propulsiva di cui ha bisogno solo dalla partecipazione democratica e dalla esaltazione dei poteri dell'assemblea rappresentativa ${ }^{79}$. Nell'articolo grande peso riveste poi il tema della battaglia contro l'installazione degli euromissili: «Non c'è partito, piccolo o grande, socialista, comunista, ecologista o cristiano che» afferma il dirigente comunista «essendosi impegnato nella lotta per la pace e contro gli euromissili non sia stato premiato dagli elettori» ${ }^{80}$. Petruccioli sottolinea quindi come la reale novità della tornata elettorale sia rappresentata dall'eclissi del vecchio europeismo e dalla vitalità di un nuovo europeismo che si colloca a sinistra:

Colpisce la fatica che registrano ovunque le forme dell'europeismo tradizionale, quasi che la dimensione europea non sia più loro congeniale. [...] Al contrario irrompono come protagonisti di un nuovo sentimento e di una nuova coscienza europea forze della sinistra ${ }^{81}$.

La riflessione sul ristagno dei vecchi modelli e l'ascesa di nuovi conflitti viene sviluppata anche nell'articolo conclusivo a firma di Giuseppe Vacca. Nel contributo, dal titolo Declino del modello neoconservatore, l'intellettuale comunista esamina quei segni che, a suo giudizio, segnano una inversione di tendenza che coinvolge tutti i Paesi europei. Per Vacca una valutazione unitaria del voto conservatore deve necessariamente tenere conto che, nel Parlamento europeo, la demarcazione fra schieramento conservatore e progressista non coincide con le forze schierate a sostegno o meno del processo di integrazione. Se da un lato l'articolo di Vacca si interroga compiutamente sul dato del calo della partecipazione al voto e dell'affievolimento della fiducia nei confronti delle istituzioni comunitarie, dall'altro pare sottovalutare la longevità di quel

\footnotetext{
${ }^{78}$ Per la ricostruzione della crisi di identità del Pci a partire dagli anni Ottanta si veda IGNAZI, Piero, Dal Pci al Pds, Bologna, Il Mulino, 1992, pp. 44-60; DI GIACOMO, Michelangela, DI NUNZIO, Novella, Trent'anni dopo. Il Pci degli anni Ottanta, Genova, Oltre, 2016.

${ }^{79}$ PETRUCCIOLI, Claudio, «Un grande elettore chiamato pace», in Rinascita, 25/1984, p. 27.

${ }^{80}$ Ibidem.

${ }^{81}$ Ibidem, p. 38.
} 
«modello neoconservatore» i cui esiti sarebbero stati evidenti negli anni Novanta ma che già nel decennio qui in esame avevano dispiegato le proprie premesse ${ }^{82}$.

A giudizio dello scrivente, se si considera la serie di elezioni italiane dalla nascita della Repubblica sino alla consultazione in esame, appare evidente come il sorpasso del Pci sulla Dc sia gravido di un forte valore simbolico. Sul tema si è più volte affermato che nel risultato elettorale abbia pesato molto la commozione per la scomparsa di Enrico Berlinguer e ciò non può che avere un fondo di verità. Tuttavia, tale analisi risulterebbe incompleta qualora non si evidenziasse come la commozione per la morte del segretario del Pci si rivestì anche di un chiaro significato politico. Vi era, in larga parte del Paese, un sincero riconoscimento del valore delle battaglie condotte da Enrico Berlinguer. Nella biografia del leader comunista si riassume infatti quella che è stata definita una "compenetrazione conflittuale tra nazionale e internazionale» che diede vita ad una strategia politica il cui studio non può esaurirsi nella storia del comunismo ma va contestualizzato nella storia d'Italia e in quella delle sinistre europee ${ }^{83}$.

A partire dal risultati elettorale del 1984, è possibile affermare che l'azione del Pci guidato da Berlinguer determinò il definitivo risanamento di quella frattura tra proletariato italiano e prospettiva europea che risaliva ai primi anni Cinquanta ${ }^{84}$. Un risultato frutto di un percorso complesso, spesso non lineare, ma legato da alcuni fili conduttori - come il tema della pace e dell'autonomia europea - che ne hanno determinato l'evoluzione nel corso di decenni caratterizzati da profondi mutamenti nel panorama delle relazioni internazionali, nella natura dell'integrazione europea e all'interno della stessa società italiana.

\section{Riflessioni conclusive}

L'analisi della stampa periodica presa in esame in relazione agli eventi storici qui presentati permette di tracciare alcune considerazioni finali.

La prima competizione europea - seppur condotta da tutte le forze politiche italiane ricorrendo ad argomentazioni più strettamente connesse alla politica nazionale che comunitaria ${ }^{85}$ - si configurò come la prima mobilitazione di tutto il Partito sul fronte europeo. Per tale ragione la consultazione del 1979 rappresentò una cesura importante nella riflessione internazionale del Partito, poiché incoraggiò un più consapevole dibattito sull'unificazione europea e sui rapporti internazionali del Pci che coinvolse, per la prima volta, anche la base del Partito. L'impegno

\footnotetext{
${ }^{82}$ COLARIZI, Simona, CRAVERI, Piero, PONS, Silvio, QUAGLIARIELLO, Gaetano (a cura di), Gli anni ottanta come storia, cit., p. 9.

${ }^{83}$ SORGONÂ, Gregorio, «Il dibattito su Berlinguer. Testimoni e storici a confronto», in Dimensioni e problemi della ricerca storica, 2/2016, pp. 11-36 (p. 33).

${ }^{84}$ MAGGIORANI, Mauro, FERRARI, Paolo, op. cit., p. 75.

${ }^{85}$ 1979: Le prime elezioni europee. Intervista a Marisa Rodano (testimonianza raccolta a Roma l'11 giugno 2003), in MAGGIORANI, Mauro, FERRARI, Paolo, op. cit., p. 209.
} 
comunitario favorì altresì il superamento di alcune rigidità ideologiche sino ad allora ancora fortemente radicate nel gruppo dirigente, ponendo nuovi interrogativi sul ruolo del movimento comunista e sui suoi rapporti con il mondo del socialismo europeo. Alla luce di questa ultima riflessione si possono identificare nel più stretto rapporto tra comunisti italiani ed Europa cesure importanti alle quali qui si è brevemente accennato, come la critica all'intervento sovietico in Afghanistan - che segnò il momento più teso tra Botteghe Oscure e Mosca - e l'adesione al Progetto Spinelli, che rappresentò il sostegno al disegno più avanzato in termini di integrazione europea. Le elezioni del 1984 si caratterizzarono infine per un forte valore simbolico dapprima per la tragica fine dell'esperienza della segreteria di Enrico Berlinguer e successivamente per lo storico sorpasso del Pci sulla Democrazia cristiana.

Al netto delle differenti e contrapposte analisi sul risultato elettorale, il voto del 1984 divenne simbolicamente momento conclusivo del lungo e contradditorio «cammino verso Occidente» dei comunisti italiani. Tuttavia, lo sgretolamento ideologico del Pci che segue alla morte di Berlinguer, pose in evidenza le aporie di quell'europeismo - «socialista, neutralista e tendenzialmente terzomondista ${ }^{86}$ - che, con la dipartita del suo principale ideatore, viene deprivato della propria forza dialettica. Questo limite, insieme alle trasformazioni che investiranno il Partito tra il 1989 e il 1991, determinerà l'adesione acritica del Pci prima, e delle sue mutazioni poi, a tutte le successive iniziative promosse nell'ambito del processo d'integrazione europea. Crollato il Muro di Berlino, l'europeismo, sostituendosi all'internazionalismo, diverrà infatti, come scrive Varsori, il «maggior punto di riferimento ideologico» della sinistra postcomunista ${ }^{87}$.

\footnotetext{
${ }^{86}$ VARSORI, Antonio, La Cenerentola d'Europa? L'Italia e l'integrazione europea dal 1947 ad oggi, Soveria Mannelli, Rubbettino, 2010, p. 313.

${ }^{87}$ Ibidem, p. 385.
} 


\section{L'AUTORE}

Calogero LANERI si è laureato in Scienze Politiche e delle Relazioni Internazionali presso l'Università degli Studi di Parma con una tesi sul Partito Comunista Italiano. Attualmente frequenta il corso di Laurea Magistrale in Scienze storiche presso l'Università di Bologna. Il suo attuale ambito di interesse è rappresentato dalla storia dei partiti politici. URL: < http://www.studistorici.com/progett/autori/\#Laneri > 